\title{
"Rendimiento académico y autoestima en estudiantes de la carrera profesional de Tecnología médica de la Universidad Nacional de Jaén"
}

"Academic performance and self-esteem in students of the Medical Technology college at the Universidad Nacional de Jaén."

${ }^{1}$ Antero Alexander Cabrera T., ${ }^{\mathrm{a}}$ Nazario Aguirre B. ${ }^{\mathrm{b}},{ }^{1}$ Alicia Alarcón S. ${ }^{\mathrm{c}}$ y ${ }^{1}$ Walter Linder Cabrera T. ${ }^{\mathrm{d}}$

\section{RESUMEN}

Este trabajo tuvo como objetivo determinar cómo se relaciona la Autoestima y el rendimiento académico en estudiantes de la carrera profesional de Tecnología Médica de la Universidad Nacional de Jaén. El total de la población de alumnos matriculados en el semestre académico 2014 II (176 estudiantes) fue evaluado. El estudio es de tipo correlacional con el propósito de medir el grado de relación que existe entre las dos variables: Autoestima y Rendimiento académico de los estudiantes. El diseño de investigación que se utilizó es transversal en el tiempo y no experimental. Para la medición de la variable autoestima se utilizó como instrumento de medición el test psicológico conformado por 20 ítems, en tanto que para el rendimiento académico se utilizó los promedios ponderados, según proporcionados por la oficina de Registros Académicos de la Universidad. Se utilizó el análisis correlacional de datos, obteniéndose una correlación positiva $(r=0.462)$ entre variables.

Palabras claves: Rendimiento académico, autoestima.

\section{ABSTRACT}

The objective of this research was to determine how self-esteem and academic achievement in the Medical Technology college at the Universidad Nacional de Jaèn. The total student population enrolled in the academic term 2014 II,( 176 students ) was evaluated. The study is correlational, as it was aimed to measure the degree of relationship between two variables: Self-esteem and Academic performance of students. The research design used is not experimental, and cross-sectional (in time). To measure self-esteem variable, a psychological test consisting of 20 items was used, whereas for academic achievement weighted averages provided by the Office of Academic records. Correlational analysis was used, in which positiva correlation between variables $(r=0.462)$ was obtained.

Keywords. academic performance, self-esteem.

\footnotetext{
${ }^{1}$ Universidad Nacional de Jaén. Cajamarca. Perú.

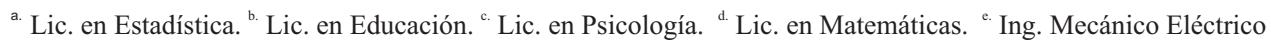




\section{INTRODUCCIÓN}

Muchos países vienen considerando el rendimiento académico y la autoestima como una relación fundamental en los procesos educativos de instituciones que tienen estudiantes que proceden de cierta etnia. En la gestión pedagógica, esta perspectiva se viene promoviendo desde algunas organizaciones, instituciones y algunos sistemas educativos, pero muy poco tiene repercusión en las aulas y en los estudiantes.

En el Perú, en las últimas décadas, el estudio del rendimiento académico relacionado con la autoestima ha sido considerado como un problema de desigualdad, marginación y opresión social. y que las formas de organización y participación de los actores educativos y de las comunidades originarias, tienen consecuencias en los aprendizajes académicos.

Por tanto, los autores creemos que es necesario que el sistema educativo y en especial la gestión pedagógica de las instituciones educativas consideren la relación entre el rendimiento académico y la autoestima como insumos indispensables en el desarrollo de los procesos pedagógicos, para tener más y mejores logros de aprendizaje, así como también garantizar una formación integral, y de calidad en los estudiantes jaenenses para desenvolverse en cualquier escenario social de la vida.

\section{MARCOTEORICO}

\subsection{RENDIMIENTO ACADÉMICO.}

La educación escolarizada es un hecho intencionado y en términos de calidad de la educación, todo proceso educativo busca permanentemente mejorar el aprovechamiento del alumno. La variable dependiente clásica en la educación escolarizada es el rendimiento o aprovechamiento escolar (Kerlinger, F. N. y Lee, H. B, 2002).
E1 rendimiento académico, también denominado rendimiento escolar, es definido por la Enciclopedia de Pedagogía / Psicología de la siguiente manera: "Del latín reddere (restituir, pagar) el rendimiento es una relación entre lo obtenido y el esfuerzo empleado para obtenerlo. Es un nivel de éxito en la escuela, en el trabajo, etc", " al hablar de rendimiento en la escuela, nos referimos al aspecto dinámico de la institución escolar. El problema del rendimiento escolar se resolverá de forma científica cuando se encuentre la relación existente entre el trabajo realizado por el maestro y los alumnos, de un lado, y la educación (es decir, la perfección intelectual y moral lograda por éstos) de otro", "al estudiar científicamente el rendimiento, es básica la consideración de los factores que intervienen en él. Por lo menos en lo que a la instrucción se refiere, existe una teoría que considera que el rendimiento escolar se debe predominantemente a la inteligencia; sin embargo, lo cierto es que ni siquiera en el aspecto intelectual del rendimiento, la inteligencia es el único factor", al analizarse el rendimiento escolar, deben valorarse los factores ambientales como la familia, la sociedad y el ambiente escolar"

Herán y Villarroel (1987) en José Leonel Nicho Alcántara 2013, sostienen que el rendimiento académico se define en forma operativa y tácita afirmando que se puede comprender el rendimiento escolar previo como el número de veces que el alumno ha repetido uno o más cursos.

Kaczynska(1986) en José Leonel Nicho Alcántara 201, afirma que el rendimiento académico es el fin de todos los esfuerzos y todas las iniciativas escolares del maestro, de los padres y de los mismos alumnos: El valor de la escuela y el maestro se juzgan por los conocimientos adquiridos por los alumnos. 
En tanto que Nováez (1986) en José Leonel Nicho Alcántara 2013, sostiene que el rendimiento académico es el quantum obtenido por el individuo en determinada actividad académica. El concepto de rendimiento está ligado al de aptitud, y sería el resultado de ésta, de factores volitivos, afectivos y emocionales, además de la ejercitación.

2.1.1 Características del rendimiento académico.

García y Palacios (1991) en José Leonel Nicho Alcántara 2013, después de realizar un análisis comparativo de diversas definiciones del rendimiento escolar, concluyen que hay un doble punto de vista, estático y dinámico, que atañe al sujeto de la educación como ser social. En general, el rendimiento escolar es caracterizado del siguiente modo:

a) El rendimiento en su aspecto dinámico responde al proceso de aprendizaje, como tal está ligado a la capacidad y esfuerzo del alumno.

b) En su aspecto estático comprende al producto del aprendizaje generado por el alumno y expresa una conducta de aprovechamiento.

c) El rendimiento está ligado a medidas de calidadyajuicios de valoración.

d) El rendimiento es un medio y no un fin en sí mismo.

e) El rendimiento está relacionado a propósitos de carácter ético que incluye expectativas económicas, lo cual hace necesario un tipo de rendimiento en función al modelo social vigente.

2.1.2 Factores generales que inciden en el Rendimiento Académico.

Muchos estudios han dado luces sobre las variables que influyen en el rendimiento académico de los estudiantes, y en lo que todos coinciden es en su naturaleza multicausal, es decir que varios factores a diferentes niveles (personales, familiares y sociales) influyen en él. Entre éstos se encuentra la variable "personalidad", que puede ser susceptible a la presión de un rendimiento académico socialmente aceptable; es decir que en la medida que un estudiante siente que no puede controlar una situación de alta exigencia no llega a desarrollar respuestas adecuadas para hacerle frente (Linn y Zippa 1984, citado en Millings, 1999). También está la ansiedad, representada por la expectativa del funcionamiento académico, y los factores sociales, tales como las relaciones interpersonales, que son estresores relacionados con el desempeño del estudiante o factores relacionados con su vida emocional (Heinz, 1984, citado por Milling, 1999) en José Leonel Nicho Alcántara 2013.

\subsection{LAAUTOESTIMA}

La autoestima es la parte evaluativa y valorativa de nosotros mismos, constituida por el conjunto de creencias y actitudes de una persona sobre sí misma, en 1981, Coopersmith corrobora sus definiciones afirmando que la autoestima es como la evaluación que una persona realiza y mantiene comúnmente sobre sí misma, se expresa a través de sus actitudes de aprobación y desaprobación, indicando el grado en que cada persona se considere capaz, significativa, competente y exitosa. Asimismo, se define a la autoestima como una actitud básica hacia uno mismo, es la forma habitual de pensar, amar y comportarse consigo. Alcantara J,L (2013).

Un adecuado nivel de auto estima es la base de la salud mental y física del organismo. El concepto que tenemos de nuestras capacidades y nuestro 
potencial no se basa sólo en nuestra forma de ser, sino también en nuestras experiencias a lo largo de la vida, Lo que nos ha pasado, las relaciones que hemos tenido con los demás (familia, amigos, maestros), las sensaciones que hemos experimentado, todo influye en nuestro carácter y por tanto en la imagen que tenemos de nosotros mismos.

La autoestima se aprende, fluctúa y la podemos mejorar. Es a partir de los 5-6 años cuando empezamos a formamos un concepto de cómo nos ven nuestros padres, maestros, compañeros y las experiencias que vamos adquiriendo, En términos sencillos podemos decir que la autoestima es la discrepancia que existe entre lo que pensamos que somos y lo que nos gustaría ser, es decir, es como valoramos lo que pensamos que somos. En el ámbito infantil o juvenil, para hacer más manejable el concepto de autoestima, podemos hablar de cinco áreas: área social (sentimientos del niño o adolescente sobre las relaciones con sus amigos), área académica (qué piensa de su faceta como estudiante), familiar (cómo se siente como parte integrante de su familia), imagen corporal (cómo ve su aspecto físico o sus capacidades físicas) y autoestima global (valoración general que hace de sí mismo). Alcantara J,L(2013)

\subsubsection{Importancia de la autoestima}

La autoestima de una persona es muy importante porque puede ser el motor que la impulse a triunfar en la vida, no en el plano económico sino en el terreno de lo personal; o hacer que se sienta verdaderamente mal aun a pesar de que parezca que lo tiene todo. Suele suceder que la imagen que los demás tienen de una persona, no guarda relación con la imagen que esa persona tiene de sí misma. En los niños sucede lo mismo. Puede llegar hasta tal punto que, cuando se convierten en adolescentes, nos encontramos con casos en los que jóvenes con buena apariencia y excelentes resultados académicos pueden, de repente, cometer intentos de suicidio porque una novia les ha dejado. Estos casos, que no dejan de sorprendernos a todos, probablemente se podrían haber evitado si ese joven hubiera tenido una alta autoestima.

Además, aunque no parece que la autoestima negativa sea la causa de importantes trastornos infantiles, sí es cierto que está presente en muchos de ellos.

La autoestima es una actitud hacia uno mismo, y éste es el modelo del cual se parte para estudiarla. Definirla como una actitud implica aceptar unos presupuestos antropológicos y psicológicos determinados, a la vez que respetar otros modelos basados en diferentes teorías de la personalidad. La autoestima como actitud es la forma habitual de pensar, amar, sentir y comportarse consigo mismo (Alcántara, J.) en José Leonel Nicho Alcántara 2013. El concepto de autoestima ha sido preocupación de muchos estudiosos; entre otros, Freud la definió como el amor propio del hombre. Otros autores han trabajado en los factores con los que se relaciona, para lograr una mayor comprensión del concepto. Así Hamacheck (1981), citado por López y Cols (1993) en José Leonel Nicho Alcántara 2013, plantea que al autoconcepto es la parte cognitiva del yo, y la autoestima es la parte afectiva del mismo, relacionándose entre sí. (López y cols.) (1993) citan otros autores que han conceptualizado sobre la autoestima: Maslow, Sears, Kaegen, Bardwick. Maslow (1962) en José Leonel Nicho Alcántara 2013, sostiene que la autoestima es el sentirse capaz de dominar algo del ambiente, saberse competente e independiente.

Kagen (1982) en José Leonel Nicho Alcántara 2013, afirma que el aprecio son valores que 
conducen al desarrollo de una autoevaluación positiva, mientras que el criticismo y el rechazo originan imágenes derrotistas que influyen en la autoestima. (Tarazona, D) expresa que la autoestima es confiar en las propias potencialidades y menciona dos componentes: la valía personal y el sentimiento de capacidad personal. La primera se refiere a la valoración positiva o negativa que la persona tiene de su autoconcepto, incluyendo las actitudes hacia sí mismo; la segunda alude a las expectativas que tiene una persona de ser capaz, de hacer de manera exitosa lo que tiene que hacer, es decir, su autoeficacia. (Labajos) establece que durante la adolescencia hay una especial preocupación por el yo, y hasta un $80 \%$ de adolescentes mantienen una imagen positiva de sí mismos, manifestando confianza en ellos; el otro $20 \%$ presenta una imagen negativa. (Montt, SM) encontró en su estudio de autoestima y salud mental, la influencia que la adecuación de la autoestima social y personal tenía con la salud mental; ésta les permitía o les impedía a los jóvenes una mejor adaptación social y emocional. La sociedad actual nos muestra que no tiene conocimientos claros para el buen desarrollo de la autoestima, y nos deja ver que sólo es consciente de a qu e 11 a s formas mínimas de socialización. Este desconocimiento afecta la práctica de estímulo que favorecen la autoestima: los cuidados, la dedicación y atención a las personas en todos los ciclos vitales, y en general todas aquellas situaciones durante la cotidianidad familiar que transmiten al individuo el mensaje de ser visible e importante para los miembros de su entorno. Walter Riso manifiesta una paradoja al expresar que nuestra civilización intenta inculcar principios como el respeto, el sacrificio, el altruismo, la expresión de amor, el buen trato, la comunicación, pero estos principios están dirigidos al cuidado de otros humanos, y se descuidan los dirigidos a nosotros mismos. Expresa este mismo autor que el auto respeto, el auto amor, la auto confianza y la auto comunicación no suelen tenerse en cuenta; más aún, se considera de mal gusto el quererse demasiado, lo cual está asociado fuertemente con nuestraestructura mental, muchas veces reflejada en nuestras expectativas. Expresa este autor: "Nos interesa más la evaluación ajena que la autoevaluación y nos hacemos víctima de nuestro propio invento, la auto insensibilidad nos ha hecho olvidar aquellas épocas de la niñez cuando todo era impactante y gratificante, estamos orientados demasiado hacia fuera, buscando la aprobación de los demás y no gastamos el tiempo suficiente en auto alagarnos y en gustarnos." La autoestima formada incide en las esferas psicoemocionales del individuo a través de su vida y condiciona la calidad de su experiencia en todas y cada una de las dimensiones de interacción con el entorno.

P. Ortega Ruiz, R. Mínguez Vallejos y M. L. Rodes Bravo. (2001), sostienen que el término autoestima es, sin duda, uno de los más ambiguos y discutidos en el ámbito de la psicología. Auto aceptación, auto ajuste, auto valoración, autoestima, auto concepto, auto imagen son términos que se usan indistintamente para significar el concepto, positivo o negativo, que uno tiene de sí mismo. Algunos autores consideran la autoestima como un constructo hipotético que representa el valor relativo que los individuos se atribuyen o que creen que los demás les atribuyen. Musitu y otros (1996), por su parte, definen la autoestima como el concepto que uno tiene de sí mismo, según unas cualidades que a sí mismos se atribuyen. No hay, por ahora, una posición unánime respecto a qué es la autoestima. Cada autor la define desde un punto de vista singular. Como dice Smelser (1989: 9): «Disponemos de una percepción bastante firme de lo que significa el término autoestima, tal y como se revela mediante nuestra propia introspección y la observación de la conducta ajena. Pero cuesta mucho expresar tal comprensión en términos precisos». 
A. Alvarez Delgado, G. Sandoval Vargas, S. Velásquez Sandoval. (2007), sostienen que: un adolescente con alta autoestima aprende más eficazmente, desarrolla relaciones más gratas, está más capacitado para aprovechar las oportunidades que se presenten para trabajar productivamente y ser auto- eficiente; comparte, invita a la integridad, siente que es importante, tiene confianza en su propia competencia, tiene fe en sus propias decisiones y en que ella misma significa su mejor recurso.

En cambio, una persona con autoestima baja, sufre en forma crónica efectos negativos, especialmente sentimientos de inferioridad. Esto hace a las personas ansiosas, depresivas, aunque investigaciones más recientes han demostrado que los individuos con autoestima baja tienden a ser más precavidos, más auto - protectores y más conservadores que arriesgados; es como una protección a la vulnerabilidad.

\section{MATERIALES Y MÉTODOS}

Debido a las características de la muestra y al problema materia de investigación, se trató de un estudio de tipo correlacional con el propósito de medir el grado de relación que existe entre dos variables, rendimiento académico y la autoestima en estudiantes de acuerdo a la definición brindada por Hernández (2010), sobre los estudios correlaciónales. El diseño de la investigación es No Experimental, La población muestra se conformó por 176 estudiantes matriculados desde el segundo hasta el sexto ciclo, en el semestre académico 2014-II de la carrera profesional de Tecnología Médica de la Universidad Nacional de Jaén, donde se considera, como unidad de análisis un estudiante.

Para conocer el rendimiento académico se utilizó los promedios ponderados, proporcionados por la oficina de registros académicos de dicha universidad y para la medición de la variable autoestima se utilizó un cuestionario aplicado por la universidad Faustino Sánchez Carrión y modificado en algunas sinonimias por los autores como instrumento dirigido a los estudiantes. Este instrumento está conformado por 20 ítems que permite evaluar y analizar dicha variable.

Para el análisis de información se usó frecuencias absolutas simples y porcentuales representadas en cuadros y gráficos estadísticos. Para el análisis de datos que permita obtener resultados sobre la relación entre la autoestima y el rendimiento académico, se utilizó el análisis correlacional, con un programa estadístico SPSS para el procesamiento de los datos.

\section{RESULTADOS Y DISCUSIÓN}

Los siguientes cuadros y gráficos muestran los resultados al aplicar los instrumentos de recolección de datos y técnicas para el análisis e interpretación de la información.

Cuadro 1. Distribución de los estudiantes de Tecnología Médica de la UNJ - Cajamarca, según niveles de autoestima.

\begin{tabular}{|c|c|c|}
\hline Nivel de autoestima & Frecuencia & Porcentaje \\
\hline $0-15$ & 35 & 21,1 \\
$16-30$ & 94 & 56,6 \\
$31-40$ & 37 & 22,3 \\
Total & 166 & 100,0 \\
\hline
\end{tabular}

Fuente: Universidad Nacional de Jaén.

Gráfico 1. Estudiantes de Tecnología Médica de la UNJ - Cajamarca, según niveles de autoestima.

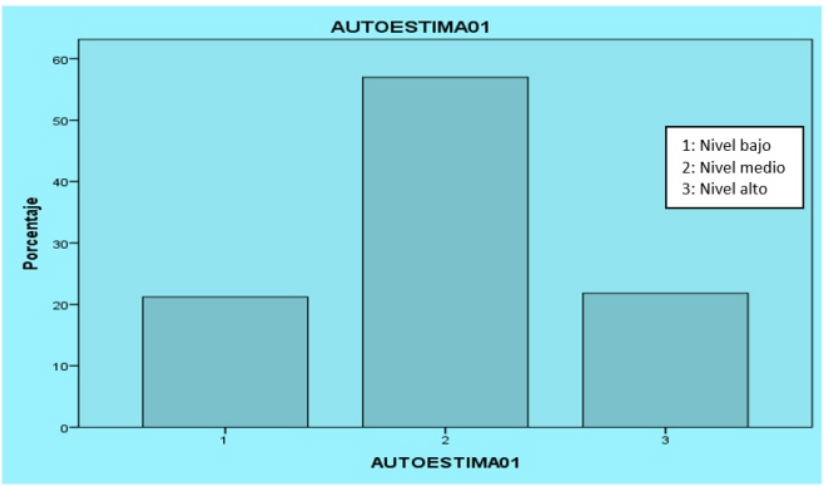

Fuente: Universidad Nacional de Jaén. 
Interpretación: En el cuadro se puede observar que el $56.6 \%$ de los estudiantes de la carrera profesional de Tecnología Médica tienen un nivel medio( $16-30)$ de autoestima, mientras que el $21.1 \%$ de los estudiantes tienen un nivel bajo( 0 - 15$)$ de autoestima.

Cuadro 2. Distribución de los estudiantes de Tecnología Médica de la UNJ - Cajamarca, según niveles de rendimiento académico.

\begin{tabular}{|c|c|c|}
\hline Rendimiento académico & Frecuencia & Porcentaje \\
\hline $0-10$ & 25 & 15,1 \\
$11-14$ & 134 & 80,7 \\
$15-17$ & 7 & 4,2 \\
Total & 166 & 100,0 \\
\hline
\end{tabular}

Fuente: Universidad Nacional de Jaén.

Gráfico 2. Estudiantes de Tecnología Médica de la UNJ - Cajamarca, según niveles de rendimiento académico.

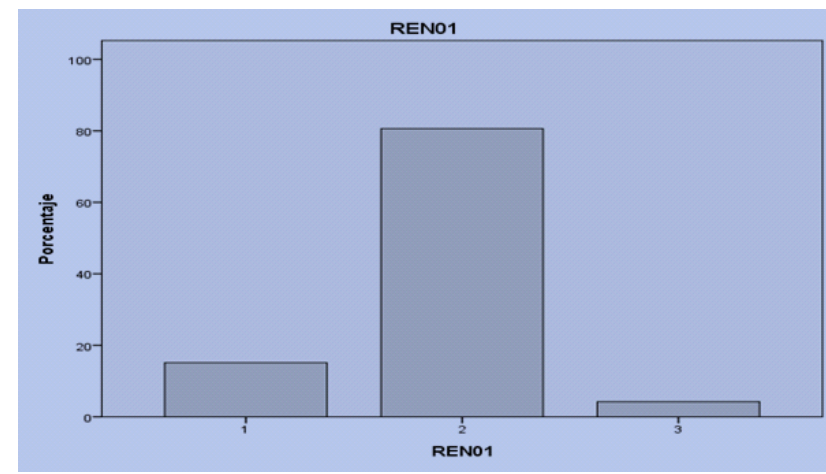

Fuente: Universidad Nacional de Jaén.

Interpretación: En el cuadro se puede observar que el $80.7 \%$ de los estudiantes de la carrera profesional de Tecnología Médica tienen un nivel de rendimiento académico regular $(11-14$ ), mientras que el $4.6 \%$ de los estudiantes tienen un nivel bueno $(15-17)$ de rendimiento académico.

Cuadro 3. Correlación del rendimiento académico en relación a la autoestima de los estudiantes en la carrera profesional Tecnología Médica de la UNJ.

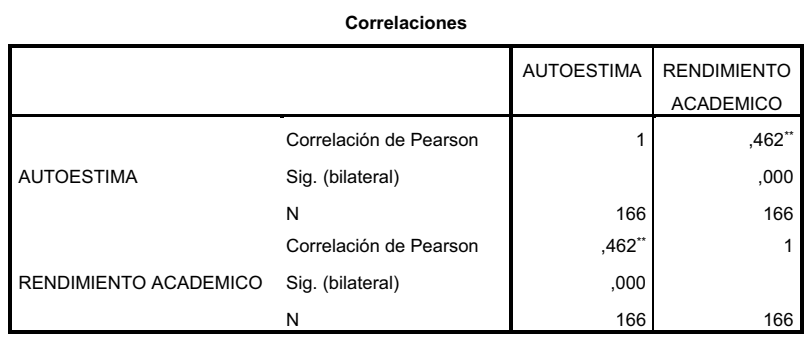

**. La correlación es significativa al nivel 0,01 (bilateral).

Gráfico 3. Correlación del rendimiento académico en relación a la autoestima de los estudiantes en la carrera profesional Tecnología Médica dela UNJ

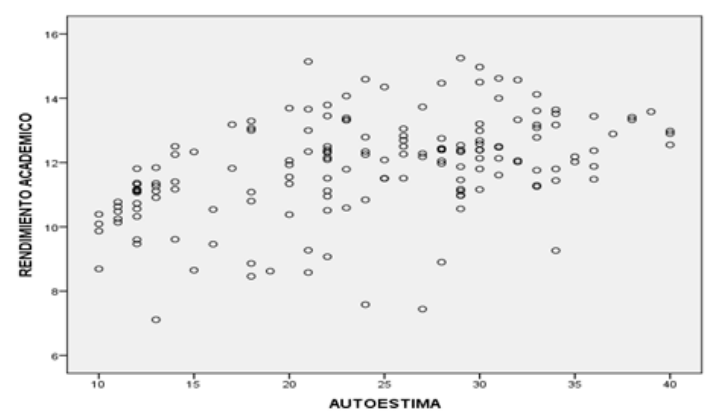

Según los resultados obtenidos (Cuadro 1) se puede afirmar que el nivel de autoestima de los estudiantes en la carrera profesional de Tecnología Médica de la UNJ, tienen un nivel medio con una puntuación de 16 a 30, que corresponde al $56.6 \%$ de la población estudiantil; mientras que el $21.1 \%$ de los estudiantes tienen un nivel bajo de autoestima con una puntuación de 0 a 15. La diferencia del porcentaje de los estudiantes con respecto del nivel medio al nivel bajo es de $35.5 \%$. Con respecto al objetivo dos sobre el nivel de rendimiento académico de los estudiantes en la carrera profesional de Tecnología Médica de la UNJ, se afirma que el $80.7 \%$ de estos estudiantes tienen un nivel de rendimiento académico regular que oscila entre 11 a 14 puntos, mientras que el $4.6 \%$ de los estudiantes tienen un nivel bueno con una puntuación de 15 a 17 puntos de rendimiento académico. La diferencia de porcentaje de los estudiantes con respecto al nivel regular y bueno es de $76.1 \%$, lo cual amerita que existe la necesidad de buscar alternativas para mejorar el nivel académico de los estudiantes a sí disminuir 
la brecha académica existente entre los niveles académicos antes mencionados. Además, en cuanto al tercer objetivo podemos mencionar que existe una asociación entre las variables, pero no es intensa, ya que se encuentra por debajo del 0.5 de la correlación de Pearson de los estudiantes en la carrera profesional Tecnología Médica de la UNJ.

\section{CONCLUSIONES}

1. Que la autoestima en los estudiantes de Tecnología Médica de la Universidad Nacional de Jaén, se concentra mayormente en el nivel medio con $56.6 \%$ de la población.

2. Que el rendimiento académico en los estudiantes de tecnología médica de la UNJ, se concentra mayormente en el nivel regular ( 11 - 14 ) con $80.7 \%$ de la población y ningún estudiante en el nivel muy bueno.

3. La correlación del rendimiento académico con la autoestima de los estudiantes en la carrera profesional Tecnología Médica es positiva con $\mathrm{r}=0.462$

\section{RECOMENDACIONES}

1. Realizar programas psicopedagógicos de autoestima académica y personal para elevar los niveles bajo y medio de autoestima, teniendo en cuenta los resultados obtenidos en este estudio con los estudiantes de Tecnología Médica de la UNJ.

2. Evaluar la autoestima cada semestre para aplicar programas psicopedagógicos a los estudiantes de Tecnología Médica que lo requieran para elevar su autoestima y rendimiento académico.

3. Realizar talleres de reforzamiento académico y equipos de estudio con estudiantes de ciclos avanzados para trasladar experiencias personales y académicas.

4. Los programas psicopedagógicos de autoestima, talleres de reforzamiento académico y equipos de estudio se realicen en todas las carreras profesionales de la UNJ.

\section{AGRADECIMIENTOS}

A los estudiantes de la carrera profesional de Tecnología Médica de la Universidad Nacional de Jaén por su colaboración en responder el cuestionario de preguntas, que brindo información real de los estudiantes UNJ.

\section{REFERENCIAS BIBLIOGRAFICAS.}

Alcántara, J. (2005). ¿ Que es la Autoestima? Como educar la autoestima. 3 a ediciòn.. españa: Ceac

Alcantara., J. L. (2013). Relación entre la Autoestima $Y$ el Rendimiento Académico de los estudiantes de la E.B.C Tecnologica de la Facultad de Educación. Perú: Universidad Nacional. Faustino Sanchez Carrión.

Esteba, E. (2004). Como desarrollar la Autoestima en el hogary la escuela. Peru: Maestro Innovador.

Hernandez R. Fernandez, C. baptista, M. (2010). Metodologia de Investigación. 5a ediciòn. Mexico: McGrawHill.

Hernández, F. (2005). Métodos y técnicas de estudio en la Universidad. Colombia: Mc Graw Hill.

Izquierdo, C. (2001). La Autoestima. . Colombia: Instituto Misionero de San Pablo.

Maddox, H. (2004). Como estudiar . España: Oikos Tau.

Meenes. (2005). Como Estudiar Para Aprender. Buenos Aires. Argentina: Paidós.

Mézerville, D. (2006). Ejes de La Salud Mental. Los procesos de autoestima, dar y recibir afecto y adaptaciónal estrés. México: Trillas.

Horna, R. (2007). Aprendiendo a Disfrutar del Estudio. Peru: Renalsa.

Ramirez, W. (2004). Autoestima y Excelencia Personal. Lima Peru: Palomino.

Santrock, J. (2007). Psicologia de la educación. México: Mc Graw Hill.

Touron, J. (2005). factores del Rendimiento Acedémico. España: Universidad de Navarra. 


\section{CORRESPONDENCIA}

Antero Alexander Cabrera Torres

Calle Húsares de Junín $N^{\circ} 146$ José Leonardo

Ortiz - Chiclayo - Lambayeque - Perú

acabreratorres@unj.edu.pe

alexander_cabrera torres@hotmail.com 